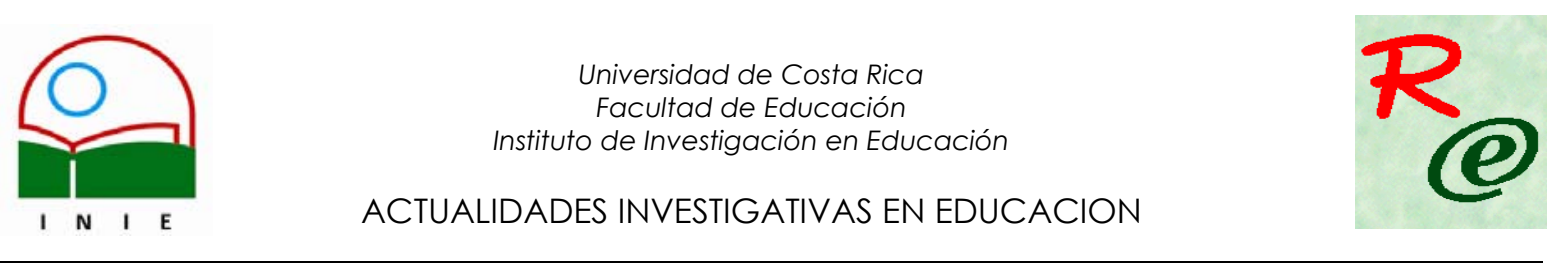

\title{
EL INSTITUTO DE INVESTIGACIÓN EN EDUCACIÓN Y LA ACCIÓN SOCIAL: AÑO 2004
}

\begin{abstract}
Julieta Castro Bonilla ${ }^{1}$
Resumen: En el presente artículo se ofrece una reflexión respecto a los antecedentes e importancia que posee la Acción Social para el Instituto de Investigación en Educación (INIE), mediante el programa Educación Continua y Servicios Especiales.

Se hace referencia al compromiso que posee la Universidad de Costa Rica como institución académica que desde su fundación, en el año 1940, se compromete con la sociedad costarricense al brindar profesiones con cualidades humanas, culturales y sociales, y que el INIE, como unidad académica adscrita a la Facultad de Educación, coadyuva con esta importante tarea al proyectar y transmitir los resultados de sus investigaciones al sistema educativo, formal e informal, mediante la organización de actividades de extensión docente.

El texto hace referencia a las actividades que durante el año 2004 se ofrecieron a la sociedad costarricense, las que permitieron el logro de los objetivos propios del programa mencionado. Se considera importante indicar que las experiencias logradas benefician el quehacer del Instituto, y lo compromete a continuar ofreciendo a la comunidad costarricense un trabajo que se caracteriza por su seriedad y calidad académica.

Palabras claves: ACCIÓN SOCIAL/INSTITUTO DE INVESTIGACIÓN EN EDUCACIÓN-INIE/ INVESTIGACIÓN/ EDUCACIÓN PREESCOLAR/ EDUCACIÓN PRIMARIA/ EDUCACIÓN SECUNDARIA/ EXTENSIÓN DOCENTE/ TECNOLOGÍA/

Abstract: In the present article, a reflection regarding the background and the importance of Social Action is offered; through the program "Continuous Education and Special Services", in the Instituto de Investigation en Educación, INIE (Institute of Research in Education). A reference is made to the commitment of the University of Costa Rica as an academic institution that, since its foundation in 1940, has become involved with Costa Rica's society by providing professions with humanitarian, cultural and social qualities; and that the INIE, as an academic unit associated to the Faculty of Education, is helping with this important task when projecting and broadcasting the results of their research to the formal and non-formal education system, through the organization of activities related to educative extension.

The text makes reference to the activities intended for the benefit of Costa Rica's society, carried out during the year of 2004; specifically those that have helped achieve the program's objectives. It is an important fact that the attained experiences have benefited the ensuing work and image of the Institute, and that they have reinforced its commitment to supply Costa Rica's community with a work that is meaningful and of academic quality.
\end{abstract}

Keywords: SOCIAL ACTION/ INSTITUTE OF INVESTIGATION IN EDUCATION/ INVESTIGATION/ PREESCOLAR EDUCATION/ PRIMARY EDUCATION/ SECONDARY EDUCATION/ EDUCATIONAL EXTENSION/ TECHNOLOGY/

\footnotetext{
${ }^{1}$ Magíster en Ciencias de la Educación con énfasis en Educación Artística. Licenciada en Bellas Artes con especialidad en Pintura. Profesora de Educación Secundaria con énfasis en Artes Plásticas. Actualmente es profesora de la Escuela de Formación Docente e Investigadora del Instituto de Investigación en Educación (INIE) de la Universidad de Costa Rica.
}

Correo electrónico: inie@cariari.ucr.ac.cr

Artículo recibido: 15 de junio, 2005

Aprobado: 29 de junio, 2005 


\section{Introducción}

El Instituto de Investigación para el Mejoramiento de la Educación CostarricenseIIMEC, se funda en febrero de 1980 bajo la denominación de Unidad Académica adscrita a la Facultad de Educación de la Universidad de Costa Rica.

Dedicado a la investigación y a la búsqueda y producción de conocimientos por medio del estudio de la teoría y de la práctica pedagógica, desde sus orígenes se plantea como meta el mejoramiento de la educación costarricense por medio de la proyección y transmisión de los resultados de sus investigaciones al sistema educativo formal e informal.

Para este Instituto, su campo de acción se encuentra claramente definido en los Principios, Propósitos y Funciones de la Universidad de Costa Rica, establecidos en el Estatuto Orgánico. A continuación de indican los que tiene relación directa con la naturaleza del artículo: la acción social en el Instituto de Investigación en Educación.

ARTíCULO 3.- El propósito de la Universidad de Costa Rica es obtener las transformaciones que la sociedad necesita para el logro del bien común, mediante una política dirigida a la consecución de una verdadera justicia social, del desarrollo integral, de la libertad plena y de la total independencia de nuestro pueblo.

ARTíCULO 4.- Para este propósito la Universidad estimulará la formación de una conciencia creativa, crítica y objetiva en los miembros de la comunidad costarricense, que permita a los sectores populares participar eficazmente en los diversos procesos de la actividad nacional. ARTÍCULO 5.- El propósito general y los objetivos inmediatos de la Universidad de Costa Rica demandan de ella la búsqueda constante, inagotable y libre, de la verdad, la eficacia y la belleza.

ARTíCULO 6.- Son funciones de la Universidad de Costa Rica:

a) Contribuir al progreso de las ciencias, las artes, las humanidades y la técnica, reafirmando su interrelación y aplicándolas al conocimiento de la realidad costarricense.

b) Formar un personal idóneo que se dedique a la enseñanza, las ciencias, las artes y las letras, para que participe eficazmente en el desarrollo del sistema de educación costarricense.

c) Contribuir a elevar el nivel cultural de la nación costarricense mediante la acción universitaria. 
En su labor investigativa, el Instituto tiene presente el bienestar social, así como las posibilidades de desarrollo económico, científico y la producción de una mentalidad democrática e igualitaria, que favorecen el progreso y la democracia. Gurdián F. (2001).

Como se indicara anteriormente, tiene como meta el mejoramiento de la educación en Costa Rica, al considerar que el sistema educativo es dinámico y se va transformando con el transcurso del tiempo, aumentando y diversificando sus necesidades de educación, así como variando su distribución en el territorio. Esto quiere decir que se expande por causa de nuevos componentes $u$ ofertas que se le agregan. De acuerdo con María Eugenia Dengo (2004), el sistema educativo crece en cuanto al factor cuantitativo y en el sentido cualitativo, ya que está obligado a evolucionar en cuanto a la modernización de sus estructuras, actualización de los contenidos programáticos y las técnicas de enseñanza, por lo que requiere de ofertas concretas de instituciones públicas como las Universidades, que coadyuvan en esa transformación, pues la educación de calidad constituye para un pueblo la mejor garantía del disfrute de sus derechos.

A 25 años de su creación, el Instituto, posee una basta experiencia en el campo de la investigación que le ha permitido acumular un importante acervo de conocimientos que le brindan la oportunidad de mantener un papel protagónico en la búsqueda de metas educativas de calidad. Cuenta con un equipo investigador de alto nivel en varias disciplinas de la educación, y por poseer un carácter interdisciplinario, enriquece su campo de acción con personal de las Escuelas que conforman la Facultad de Educación, así como de otras unidades académicas de la Universidad.

El Instituto tiene claro que debe hacer sus mejores esfuerzos por mejorar y mantener su calidad investigativa mediante procesos que le permitan una constante renovación de su labor diaria. En la actualidad se encuentra conformado por los siguientes programas:

- Mejoramiento de los Procesos de Enseñanza y Aprendizaje

- Investigación en tecnologías de la información y la Comunicación

- Investigación en Educación y Género

- Cambio, Desarrollo y Gestión de la Educación Superior

- Educación Continua y Servicios Especiales

- Educación y Cultura (vigente a partir del año 2006).

Cada uno de éstos fortalece su quehacer investigativo y produce conocimientos enmarcados por ejes transversales que son la razón de ser del Instituto. Acorde con el nombre del artículo, me referiré únicamente a la labor desarrollada por el Programa 
Educación Continua y Servicios Especiales, el cual se encuentra adscrito a la Vicerrectoría de Acción Social y cumple con el propósito de relacionar los resultados de sus investigaciones con la acción social de la Universidad de Costa Rica, programando actividades de extensión docente a diversas poblaciones de la sociedad costarricense.

\section{Fundamentación Teórica}

El Instituto de Investigación en Educación-INIE-, y el programa Educación Continua y Servicios Especiales, fundamentan su marco de acción en los siguientes postulados:

\section{- Fines de la educación costarricense}

- La formación de ciudadanos amantes de su Patria, conscientes de sus deberes y sus derechos y de sus libertades fundamentales, con profundo sentido de responsabilidad y de respeto a la dignidad humana.

- Contribuir al desenvolvimiento pleno de la personalidad humana.

- Formar ciudadanos en una democracia en la que se conciben los intereses del individuo con los de la comunidad.

- Estimular el desarrollo de la solidaridad y la comprensión humana

- Conservar y ampliar la herencia cultural, impartiendo conocimientos sobre la historia del hombre, las grandes obras de literatura y los conceptos filosóficos fundamentales.

Es importante indicar que los Fines de la Educación Costarricense definen nuestra labor y nuestra razón de ser, pues presentan una orientación clara respecto al ideal de hombre que la sociedad costarricense quiere formar, por lo tanto, el Instituto tiene presente que en la formulación de sus proyectos investigativos y de sus actividades de extensión docente no podemos perderlos de vista:

\section{- Misión de la Universidad de Costa Rica estipulada en el Estatuto Orgánico}

Los principios de la Institución se refieren a que,

- La Universidad de Costa Rica es una institución autónoma de cultura superior constituida por una comunidad de profesores, estudiantes y funcionarios administrativos, dedicada a la enseñanza, la investigación, la acción social, el estudio, la meditación, la creación artística y la difusión del conocimiento. 
- La Universidad de Costa Rica goza de independencia para el desarrollo de sus funciones y de plena capacidad jurídica para adquirir derechos y contraer obligaciones, así como para darse su organización y gobierno propios. Su régimen decisorio es democrático y por consiguiente en ella las decisiones personales y colectivas se realizan con absoluta libertad.

Son funciones de la Universidad de Costa Rica:

- Artículo 6. Contribuir al progreso de las ciencias, las artes las humanidades y la técnica, reafirmando su interrelación y aplicándolas al conocimiento de la realidad costarricense.

\section{- Visión del INIE}

En el año 2001, el Instituto de Investigación en Educación se dio a la tarea de aplicar el FODA, o sea, un proceso evaluativo para determinar las Fortalezas, las Oportunidades, las Debilidades y las Amenazas del Instituto.

Este proceso se organizó de manera participativa, con la Decana de la Facultad de Educación y los directores de las unidades académicas de la Escuela de Formación Docente, Escuela de Administración Educativa, Escuela de Orientación y Educación Especial, Escuela de Ciencias del Deporte y la Escuela de Bibliotecología y Ciencias de la Información. También participaron en esta actividad sus ex directores, su personal investigativo y administrativo, así como representantes de la Vicerrectoría de Investigación.

Como resultado de este proceso se establece la Visión, Misión y las Políticas de los programas que delinean al instituto, así como el Plan Estratégico a desarrollar durante los siguientes ocho años, esto le permitiría organizar su labor investigativa desde un planteamiento científico, sistemático y coherente con las normas universitarias.

A continuación se hace referencia a los resultados de esos procesos interactivos:

Visión: Se considera que será una Unidad Académica de Investigación:

- Líder nacional en el campo de la investigación educativa costarricense y con proyección internacional.

- Vinculada estrechamente a los programas de posgrado y a las unidades académicas de la Facultad de Educación y otras unidades institucionales y extrauniversitarias, como promotora, inspiradora y rectora de la institución Inter. y transdisciplinaria sobre los sistemas y procesos educativos formales, no formales y a distancia. 
- Vinculada con el avance del conocimiento (generación, desarrollo, divulgación, integración y evaluación) en el campo educativo, en los ámbitos nacional e internacional, mediante un clima de innovación crítica que facilite el desarrollo humano equitativo.

- Que coadyuva en la solución de problemas del sector educativo costarricense formal, no formal y a distancia.

- Reconocida nacional e internacionalmente por su proyección mediante proyectos de investigación comparada, cooperación internacional y en la producción de tecnologías de la información y comunicación

- Con la organización flexible y un equipo de académicos y académicas de reconocida trayectoria, que promueven del desarrollo humano.

Misión: EI INIE es una Unidad Académica de Investigación que realiza investigación interdisciplinaria en educación formal e informal; que constituye soluciones transformadoras, creativas e innovadoras a la problemática existente, sustentadas en la generación de conocimiento y desarrolladas mediante una comunidad de investigadores de distintas unidades académicas de la Universidad de Costa Rica y de otras organizaciones, que asumen sus labores con profesionalismo y ética.

Las Políticas que corresponden al área de Acción Social, mediante el programa Educación Continua y Servicios Especiales, se concretaron de la siguiente manera:

- Propiciar que los programas y proyectos de investigación contemplen la divulgación de los resultados entre los públicos beneficiarios.

- Promover una cultura de publicación y divulgación de los procesos investigativos que se desarrollan en el Instituto.

- Elaborar en coordinación con las Escuelas de la Facultad de Educación, proyectos de capacitación y actualización con los resultados de sus investigaciones.

Los postulados indicados anteriormente, a saber, los Fines de la Educación Costarricense, la Misión de la Universidad de Costa Rica estipulada en el Estatuto Orgánico, la Visión y la Misión correspondientes al INIE, así como las políticas que orientan el programa de Acción Social, fundamentan el marco de acción del Instituto y son considerados el punto de partida para establecer nuestras propuestas investigativas y las actividades de extensión docente que se programan, pues inciden directamente en nuestro trabajo cotidiano. 
Además de la concepción filosófica que sustenta el concepto de educación en el Instituto, nuestro trabajo se orienta en función del desarrollo pleno, integral y armonioso de los individuos. Por lo que se considera fundamental participar en una formación humanística que enfatice la solidaridad y la cooperación, el respeto a la diversidad y a los derechos de las personas, así como a las expresiones culturales, una formación que fomente la acción responsable frente a la naturaleza y al entorno social y cultural, y que enfatice las cualidades humanas frente al vertiginoso avance científico y tecnológico. La visión humanista que posee el programa responde al planteamiento filosófico de la Universidad de Costa Rica, al considerar su permanente interés por la cultura general y humanística. (Estatuto Orgánico de la Universidad de Costa Rica, 2000).

El concepto de educación indicado anteriormente, es visualizado por uno de los gestores de la Universidad de Costa Rica, Don Rodrigo Facio, al considerar que la formación universitaria:

Está inspirada por un ideal pedagógico que no es tanto enseñar, como enseñar a aprender, no es tanto decir lo que hay que pensar, como inducir a pensar. Formar un técnico sobre el hombre de ciencia y el hombre de ciencia sobre el hombre culto, moral y socialmente responsable ${ }^{2}$.

Por consiguiente, los conceptos de educación y de extensión docente que impulsa el INIE, se convierten en agentes activadores de la conciencia social, que permiten concretar las aspiraciones de nuestra sociedad y promueven la paz y la cultura sobre una base esencialmente humanista, con especial énfasis en una educación que favorezca la calidad de vida de sus ciudadanos. De esta manera, el INIE contribuye con la educación formal y no formal ofreciendo conocimientos y experiencias concretas que surgen de los procesos investigativos producidos por su personal académico, mediante talleres, capacitaciones, asesorías y conferencias a diversas instituciones, me refiero al Ministerio de Educación Pública y a diversas instituciones y organismos nacionales e internacionales.

El Instituto ha producido en sus 25 años de existencia una importante cantidad de investigaciones que evidencian estudios de calidad en las siguientes áreas:

- Los procesos de enseñanza y aprendizaje en la educación preescolar, primaria y secundaria, y en diversas disciplinas del currículum escolar como matemáticas, ciencias, expresión artística, filosofía, español, estudios sociales, inglés, entre otras.

- Las tecnologías de la comunicación y su incidencia en los procesos educativos.

\footnotetext{
${ }^{2}$ Tomado de la inscripción de una placa ubicada dentro del campus universitario Rodrigo Facio.
} 
- La educación y la equidad de género.

- Experiencias que impulsan el cambio en instancias de educación superior

Las experiencias investigativas q ue surgen de los programas mencionados, le han permitido conformar una sólida base científica mediante la cual genera de manera constante y sistemática conocimientos y experiencias que posteriormente son ofrecidos a diversos sectores de la población.

Conocedores del acelerado desarrollo científico y tecnológico en los campos de la educación y la comunicación, el INIE -mediante la planificación de investigaciones- se convierte en el recurso estratégico que le permite a las comunidades aspirar a un mejor desarrollo. Como respuesta a las demandas del presente, los Estados formulan políticas de modernización que involucran necesariamente a las universidades y requieren de éstas el desarrollo de nuevas áreas del saber, por este motivo el Instituto se encuentra en una constante búsqueda de conocimientos con el propósito de brindar a la sociedad costarricense conocimientos de acuerdo con los requerimientos de los tiempos. (López, 2004).

\section{El desarrollo humano es un proceso continuo e integral}

De acuerdo con la información que nos brinda el Estado de la Nación, el Instituto tiene presente que el desarrollo humano es un proceso continuo e integral, que reúne componentes y dimensiones del desarrollo de las personas y de las sociedades, en el que lo más importante es generar conocimientos dentro de contextos de mayor equidad. Por lo tanto, este concepto de desarrollo obliga a las autoridades tomar decisiones de carácter integral y de largo plazo, como una forma de prever sus consecuencias en el futuro.

Acorde con ese pronunciamiento, el INIE planifica y organiza sus actividades procurando que este desarrollo se planifique desde un planteamiento continuo e integral.

Continuo: porque nunca termina, no tiene punto final de llegada, no se sabe cuándo terminará el proceso de evolución del ser humano y su sociedad, ya que conforme la humanidad avanza en el tiempo, las demandas y necesidades superadas generan nuevas necesidades y demanda, en una historia sin fin.

Integral: porque el desarrollo humano integra las principales dimensiones del desarrollo, como la económica, la sociocultural y la ambiental. Así, por ejemplo no puede considerarse un verdadero desarrollo de las personas si una sociedad acumula mucho dinero pero, junto con ello, hay mayor desempleo y mayor cantidad de gente viviendo en condiciones 
miserables; si hay universidades y colegios, pero cada vez hay menos personas que pueden estudiar en esos centros; si hay grandes avances y tecnologías de salud, pero cada vez menos gente tiene acceso a ellas; si cada vez hay más producción de bienes, pero a costa de un deterioro mayor del medio ambiente (Estado de la Nación, 2001).

El eje central del desarrollo de las personas es lograr que se generen oportunidades de crecimiento individual y colectivo de forma equitativa, lo cual quiere decir condiciones equiparables de acceso a las oportunidades para todos los grupos sociales. Se promueve la solidaridad con las generaciones futuras, al preocuparse por heredarles una tierra con recursos necesarios para el sostenimiento de la vida humana y de las formas de vida del planeta; pero también procura fomentar la solidaridad en la actual generación (Estado de la Nación, 2001, p. 16).

De acuerdo con lo expuesto, el Instituto tiene muy clara su función, al permanecer en una constante búsqueda de conocimientos que se generan a partir de la investigación educativa, en los ámbitos nacional e internacional, mediante un clima de innovación crítica. Además, coadyuva en la solución de problemas en el sector educativo costarricense formal, no formal y a distancia, mediante la organización de talleres, congresos, conferencias, ya que cuenta con un equipo de académicos-investigadores de reconocida trayectoria que promueven del desarrollo humano.

A continuación se exponen las actividades de extensión docente que el INIE organizara durante el año 2004, las cuales se ofrecieron a la sociedad costarricense en general. En éstas se indica la persona responsable de su organización, además se menciona el público que se favoreció con la actividad.

\section{Actividades de Extensión Docente desarrolladas por el Instituto de Investigación en Educación (INIE) durante en año 2004}

4.1 Estrategias no tradicionales en la enseñanza de la matemática; geometría. Dos talleres dirigidos a docentes de Educación Primaria de las Escuelas de Excelencia y PROMECUN.

Responsables de esta actividad M.Ed. Teresita Peralta Monge y M.Ed. Mario Murillo Chaves.

Los talleres "Estrategias no tradicionales en la enseñanza de la matemática; geometría", surgen de los resultados del proyecto de investigación "Construcción de conceptos geométricos en maestros de escuela primaria", realizado por los profesores Mario 
Murillo Chaves y Teresita Peralta Monge. La investigación se llevó a cabo en el INIE con el propósito de conocer los significados (que poseen los maestros) sobre los conceptos geométricos contenidos en los programas de matemática de la escuela primaria, pues se consideraba necesario contar con elementos teóricos que orientaran la construcción de material didáctico y que sirviera de apoyo para los procesos de formación y capacitación de los maestros en el área de Geometría. Como resultado de esta investigación se encontraron en los docentes deficiencias en su formación, relacionadas con:

- Los procesos de construcción geométrica.

- Uso de instrumentos geométricos.

- Abordaje por separado del estudio de los diferentes polígonos, sin considerar las relaciones existentes entre éstos.

- Ausencia de análisis de las características de los polígonos, para una construcción de relaciones matemáticas.

- Distorsiones en el abordaje de conceptos geométricos relacionados con longitud, perímetro y área.

- Manejo memorístico y automatizado de las fórmulas para el cálculo de áreas, sin atender las relaciones existentes entre estas.

- Carencias en el uso de estrategias para el cálculo de áreas de polígonos irregulares, diferentes a los convencionales.

Las deficiencias encontradas como parte de los resultados de la investigación justifican la ejecución de procesos de capacitación en geometría a maestros del I y II ciclos de la Educación General Básica, lo que les permite explorar y plantear conjeturas, analizar para encontrar y descubrir propiedades, descubrir patrones a través de la comparación, determinar relaciones matemáticas a partir de los patrones y finalmente generalizar, como un medio para el desarrollo del pensamiento.

4.2 Producción de Módulos de Capacitación Multimediales: la inclusión del niño y la niña con necesidades educativas en el campo de la lecto-escritura en el aula preescolar.

Responsable de la actividad M.Ed. José Zúñiga Sánchez.

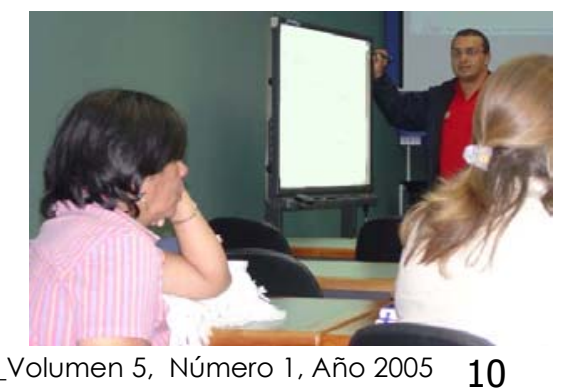


Este taller de capacitación estuvo dirigido a docentes de educación preescolar y propuso un modelo interactivo multimedial. Los conocimientos adquiridos por los participantes les permitió construir un modelo de guión multimedial, que incluyó el desarrollo de un mapa conceptual que diera como resultado una "interfaz de un producto multimedia". Como parte del taller se realizó un disco compacto interactivo con prácticas pedagógicas referente de la inclusión de la niña y el niño en el aula, el cual se encuentra en su etapa evaluativa y concluirá durante el año 2005.

La adquisición de conocimientos y habilidades respecto a la producción multimedial y su aplicación en el aula, facilitará una enseñanza apoyada en herramientas digitales audiovisuales, que estimula el desarrollo de los sentidos, lo que permite un aprendizaje significativo en el campo de la lecto-escritura, en una población estudiantil con necesidades educativas especiales.

Los conocimientos y las experiencias adquiridas por quienes participaron permitieron intercambios y experiencias didácticas enriquecedoras, que fortalecieron un trabajo de capacitación interactivo, en el que prevalecieron aprendizajes que motivaron procesos de reflexión y retroalimentación.

4.3 Jornadas de Investigación-Actividades Pre-congreso, correspondientes al "Congreso Internacional de Investigación Educativa: Investigar para transformar los procesos educativo que tuvieron lugar en el mes de enero del 2005".

Responsable de la organización de las Jornadas: M.Ed. Julieta Castro Bonilla.

Las "Jornadas de investigación-actividades precongreso", realizadas los días 11,12 y 13 de octubre del 2004, estuvieron organizadas por los coordinadores de los programas que conforman el quehacer del Instituto. A continuación se expone un resumen de cada una.

Cabe destacar que la Licenciada Carmen Cubero, coordinadora del programa: Mejoramiento de los

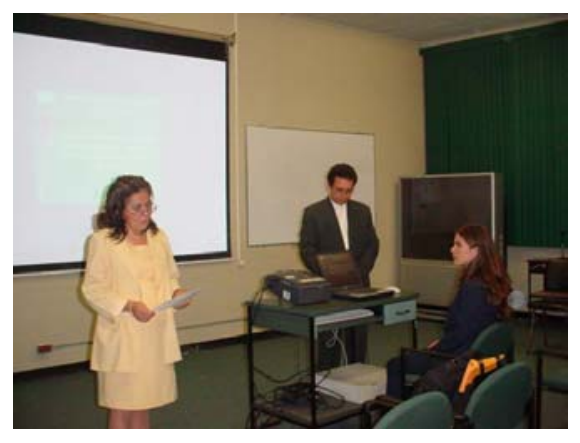

Procesos de Enseñanza y Aprendizaje contactó a la Doctora Manuela Romo, catedrática de la Universidad de Salamanca para que impartiera a la comunidad educativa dos cursos de extensión docente. Un taller denominado "Creatividad en el Aula", y una conferencia sobre el tema "Creatividad y Educación". 
- El taller "Creatividad en el Aula" contó con la presencia de docentes de educación preescolar, primaria, secundaria y universitaria, por lo que se vivieron importantes procesos de interacción dentro del contexto de aula. De acuerdo con los comentarios externados por los participantes; los conocimientos y las experiencias adquiridas, enriquecieron su bagaje académico, pues redunda en una mejor aplicación del concepto "creatividad", fundamental durante la etapa de planificación de los procesos de enseñanza y aprendizaje.

- La conferencia "Creatividad y Educación", presentó un contenido novedoso y actualizado respecto a la importancia de incrementar la creatividad en los estudiantes. El auditorio de la Facultad de Educación, lugar donde ésta se llevo cabo, estuvo muy concurrido, los asistentes tuvieron la oportunidad de participar formulando preguntas, reflexiones y comentarios.

- Otro aspecto digno de destacarse es la "Renovación del convenio entre la Universidad de Costa Rica y la Universidad Autónoma de Madrid “. Esto es importante para el Instituto pues en la formulación de su plan estratégico se encuentra la proyección internacional del mismo, así como las posibilidades de intercambio con otras instituciones de educación superior.

La Doctora Jacqueline García, coordinadora del programa: Investigación en tecnologías de la información y la comunicación, organizó la actividad denominada "Desafíos éticos y educativos de las tecnologías de la información y la comunicación”, dos mesas redondas con los siguientes temas:

- Plataformas virtuales y educación.

- Ética y tecnologías de la información y la comunicación.

Las actividades se dirigieron a profesores universitarios, investigadores, estudiantes y público interesado en tópicos relacionados con las tecnologías de la información y la comunicación. Se reflexionó sobre el papel de la tecnología educativa y su impacto en la cultura y en la sociedad en la que nos desenvolvemos.

Se dispuso en el sitio WEB del INIE (http://www.inie.ucr.ac.cr), y en la página del "Programa de Investigación sobre Tecnologías de la Información y la Comunicación", un vínculo que contiene las ponencias presentadas. 
Al contar con la participación de especialistas en las áreas de: educación, tecnologías de la información y de la comunicación, computación, filosofía y derecho, el aporte que ofreció cada exponente fue pertinente y adecuado a las expectativas de la audiencia, lo que permitió espacios reflexivos respecto a los problemas éticos de las sociedades contemporáneas, así como al papel de las tecnologías de la información y la comunicación en el campo educativo, a través de las plataformas virtuales.

Se analizó la función de las tecnologías, en relación con los problemas éticos y su aplicación en el campo educativo, de la misma manera que se fortaleció la imagen del Instituto como promotor de este tipo de actividades académica.

EI Programa de Investigación en Educación y Género-PIEG, coordinado por la Magíster Ana Lucía Villarreal Montoya, organizó un taller denominado "De la Experiencia a la Esperanza". La realización de éste permitió entre los concurrentes, espacios que motivaron los siguientes logros:

- Mediante la realización del taller se crearon espacios que motivaron la reconstrucción y transformación de procesos educativos con equidad.

- Se realizó y evaluó la programación del

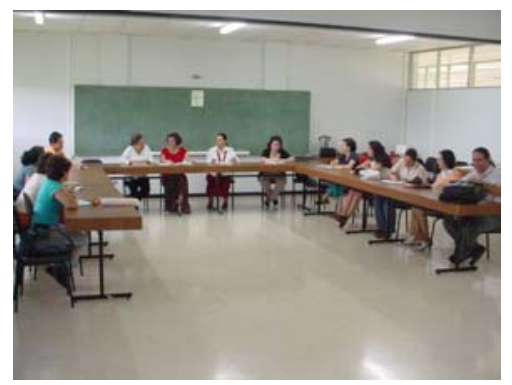
taller a partir de resultados y experiencias de proyectos de investigación que el mismo programa organiza.

- Se entregó un material escrito, que contiene los resultados de diferentes proyectos de investigación del PIEG. Los mismos se pueden utilizar en procesos de reconstrucción y transformación de aspectos educativos, dentro de la

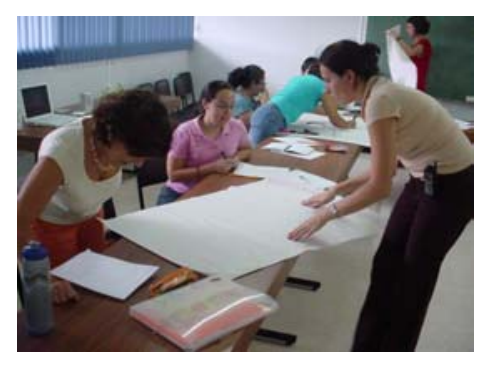
perspectiva de género y de respeto a los derechos humanos. 
El Licenciado Warner Cascante a nombre del programa: Gestión y Desarrollo de la Educación Superior, expuso una conferencia denominada "Perspectiva de un muevo modelo de competencia para los puestos de Dirección de la Universidad de Costa Rica". En ésta se expusieron los resultados de una investigación que el señor Cascante realiza, los cuales se refieren a una base de datos que ha

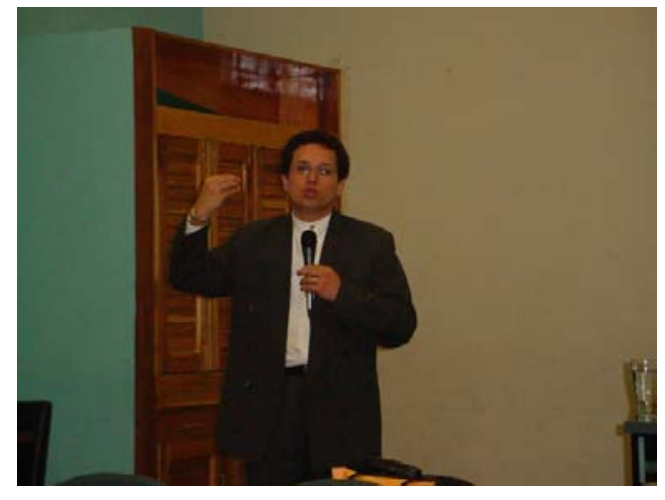
elaborado en el programa File Maker, para lo que ha tomado en cuenta los Directores de las Unidades Académicas de la Universidad de Costa Rica, así como su período de nombramiento.

Se comentó respecto a la importancia de recopilar este tipo de bibliografía con el propósito de establecer información para caracterizar a los Directores de las diversas Unidades Académicas como población de estudio, y de esta manera diseñar necesidades de capacitación.

Informó acerca de las facultades y atribuciones que el marco constitucional, legal y reglamentario establece para un Director de Escuela, y finalmente se refirió a la confección de un listado de conocimientos, habilidades y actitudes que permiten determinar lineamientos concretos de capacitación a los Directores que la requieran.

Para concluir la actividad, el señor Cascante realizó un comentario de su artículo denominado "Apuntes sobre la utilidad de un modelo de competencias aplicado a los puestos de dirección en la universidad pública costarricense", escrito para la UNESCO y publicado por la Revista Electrónica del INIE: "Actualidades Investigativas en Educación

\subsection{Taller: Educación y Tecnologías: De lo excepcional a lo cotidiano.}

Responsable de la actividad: Dra. Jacqueline García Fallas.

El taller, "Educación y Tecnologías: De lo excepcional a lo cotidiano", estuvo organizado por la coordinadora del programa Tecnologías de la Comunicación y la Información del INIE, en conjunto con la Facultad de Educación de la Universidad Estatal a Distancia (UNED) y la Fundación Omar Dengo.

En éste se compartieron las experiencias que el campo de las tecnologías y de la comunicación, han experimentado las universidades estatales y la Fundación Omar Dengo. 
La actividad permitió compartir experiencias sobre el desarrollo de las tecnologías de la información y la comunicación (TIC's), aplicadas por la Fundación Omar Dengo (en los niveles educativos de la educación general básica y diversificada), de la misma manera que en la educación superior: Universidad Estatal a Distancia, Universidad Nacional, Instituto Tecnológico de Costa Rica y la Universidad de

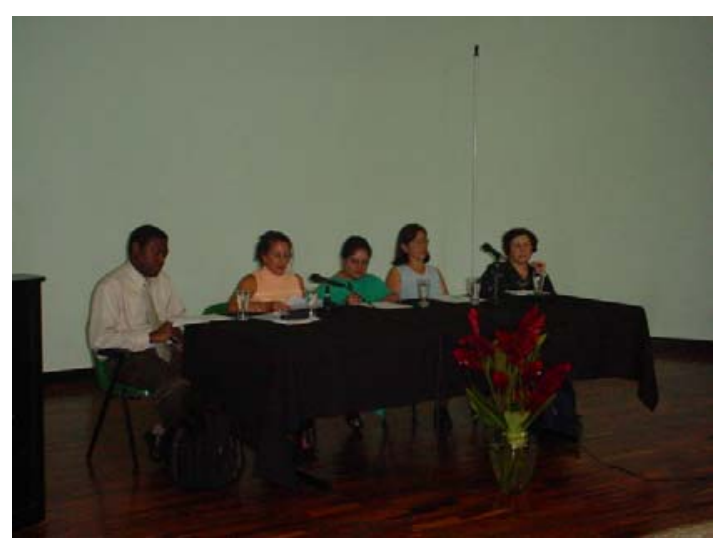
Cota Rica.

Estas experiencias se relacionaron con el desarrollo de las TIC's en el contexto español, pues el experto Antonio Bartolomé de la Universidad de Barcelona, quien presentara una ponencia, favoreció un clima de discusión participativa y académica a partir de su exposición. Los aportes teórico-metodológicos permitieron reflexionar sobre la experiencia construida en nuestro país alrededor de las Tecnologías de la Información y de la Comunicación.

4.5 Proyecto de Literatura, enlace: Hogar-Escuela de Platanares de Moravia. Responsable de la actividad: M.Ed. Julieta Castro Bonilla.

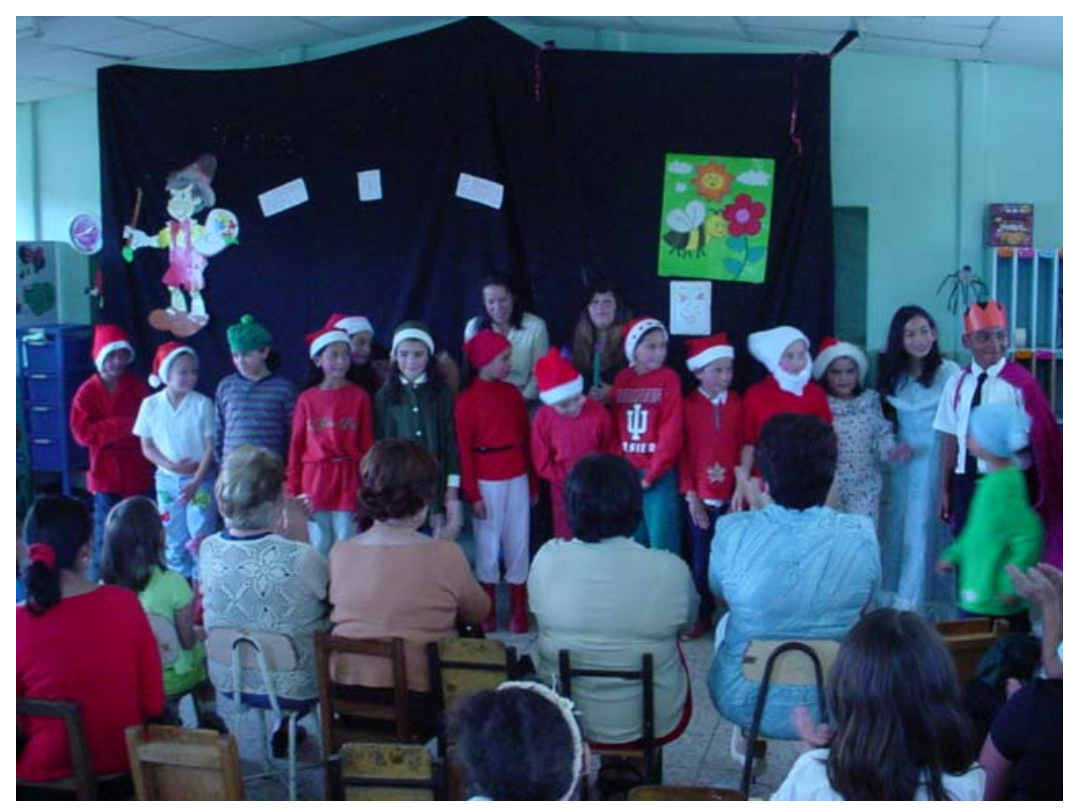


Actividad académica con una duración de ocho meses, mediante la cual se elaboró un diagnóstico dirigido a 27 padres y madres de familia, y 28 educandos del tercer grado de la Educación General Básica de la Escuela de Platanares de Moravia, que permitió la identificación de hábitos, percepciones e intereses hacia la literatura. De acuerdo con la información suministrada, se organizaron actividades académicas en las que participaron las siguientes poblaciones: maestras, familiares, integrantes del Capítulo Gamma y docentes de la Facultad de Educación de la Universidad de Costa Rica.

El concepto metodológico aplicado para enseñar la poesía, requirió de la puesta en práctica de otras áreas expresivas, como la música, las artes plásticas y la expresión corporal, por lo que se contactó con especialistas en esos temas, como los docentes Consuelo Arguedas Quesada y Carlos Rubio Torres.

Como parte de las actividades programadas, durante la última sesión, los padres de familia y estudiantes, organizaron actividades en las que llevaron a la práctica, y desde un planteamiento lúdico e integral, lo aprendido en los diferentes talleres.

Es importante mencionar que el interés por la lectura quedó manifiesto mediante la participación sistemática de los estudiantes, docentes y familiares en las diversas actividades, ya que el proyecto culminó con una producción creativa de los participantes, lo que permitió apreciar su alto grado de compromiso, dedicación y empeño.

4.6 Producción y distribución de Materiales Didácticos para la enseñanza del cálculo II MA-1002.

\section{Responsables de la actividad: Dra. Sonia Rodríguez Soto y Lic. Alberto Soto Aguilar}

Este interesante proyecto relacionó de manera integrada las áreas de Acción Social, Docencia e Investigación, al poner al servicio de 17 docentes y 1300 estudiantes del curso MA-1002, Cálculo II, de las Sedes Rodrigo Facio y Occidente, la producción y distribución de un material elaborado por los investigadores, mediante el proyecto de investigación 
denominado "Red ProEd". Como apoyo a la labor docente se crearon presentaciones para proyectarse durante las lecciones y se introdujeron softwares (WinPlot, MuPad.), que facilitaron la presentación de los conceptos y la ejercitación de éstos durante la práctica.

La compra de materiales como, 3 computadoras, un proyector y la entrega de discos compactos a los estudiantes del curso mencionado, permitieron una mayor comprensión de los temas desarrollados. Por lo tanto, se espera que a los profesores se les facilite su trabajo de aula.

Se considera importante indicar que desde la implementación del material se ha notado un aumento en el nivel de comprensión y aprobación de los contenidos del curso mencionado.

4.7 Capacitación a docentes de preescolar en torno al programa de Matemática activa y creativa.

Responsable de la actividad: Lic. María Marta Camacho Álvarez.

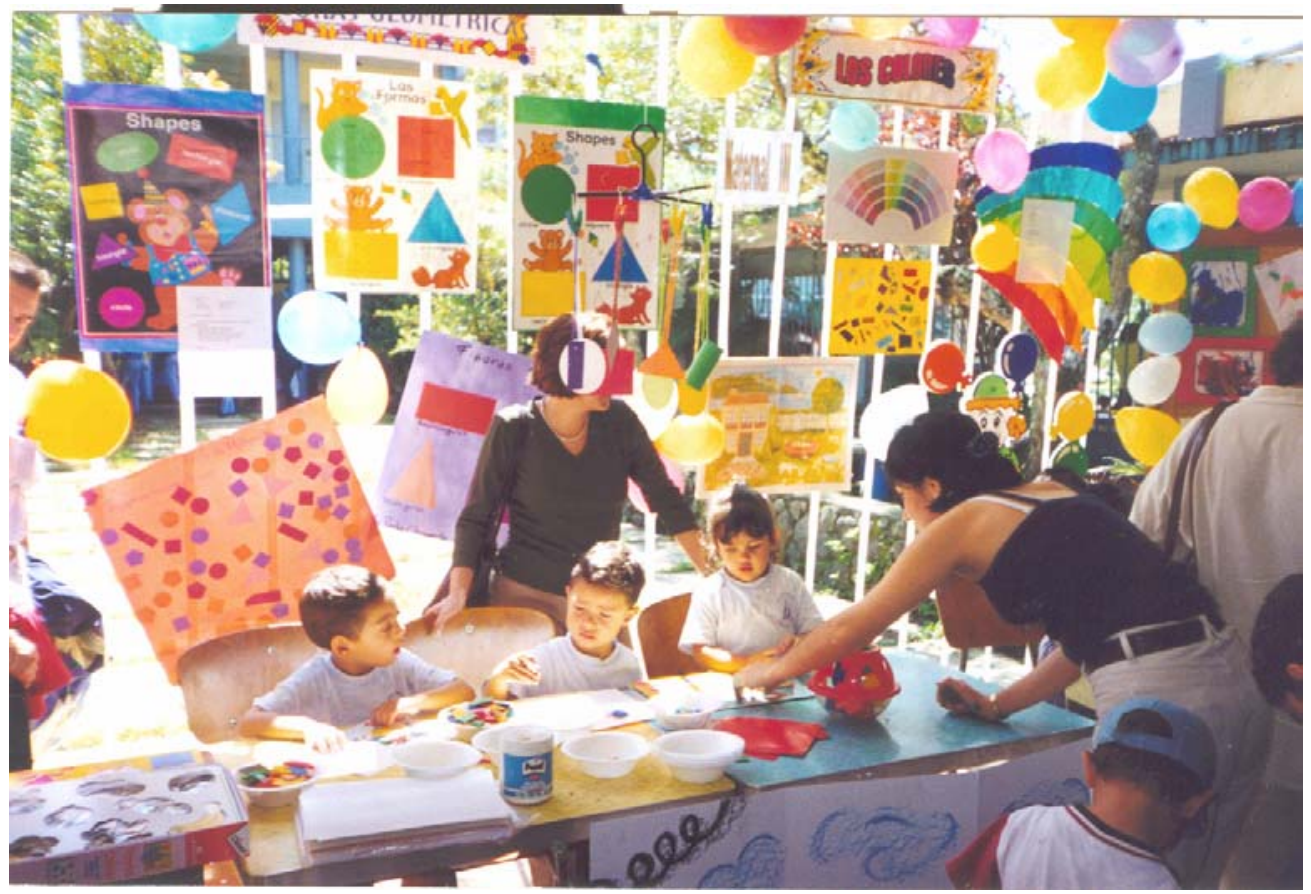

Esta actividad de extensión docente surge de la necesidad de solventar carencias en el campo de la formación docente, específicamente en el área de las matemáticas.

Se trata de una posibilidad que le permite a los docentes la actualización en este campo de estudio, y de esta manera atender con mayor eficacia los procesos de enseñanza y aprendizaje de la matemática. Pretende además, apoyar los esfuerzos que hace el INIE en 
cuanto a ofrecer programas de educación continua, así como servicios académicos a quienes atienden poblaciones en edad preescolar. El proyecto se sustenta en los resultados de una investigación denominada "El proceso de enseñanza-aprendizaje de la matemática en preescolar: visto desde la formación docente, que se desarrolla en este Instituto, así como en el programa "Matemática activa y creativa, que desde hace nueve años desarrolla la misma investigadora. El proyecto de extensión docente también de fundamenta en la experiencia de los maestros participantes, y de la población estudiantil de instituciones públicas y privadas del sistema educativo costarricense. Cabe destacar que se evidenciaron los siguientes logros:

- Proceso de capacitación a docentes de educación preescolar de las siguientes regiones:

San José, Turrialba, Heredia, Limón, San Carlos, Cartago, Palmares y Alajuela.

- Se desarrolló un curso por videoconferencia en coordinación con la Universidad Estatal a

Distancia, que culminó con el otorgamiento de Certificados a 113 docentes.

- Se realizó un "Encuentro de Matemática Activa y Creativa", en el que participaron 70 niños y niñas de 4 centros de educación preescolar, así como sus padres, madres, abuelos y abuelas.

- Se realizó un Encuentro de Educadores, en el que participaron representantes del Ministerio de Educación Pública, Universidad Estatal a Distancia y la Universidad de Costa Rica.

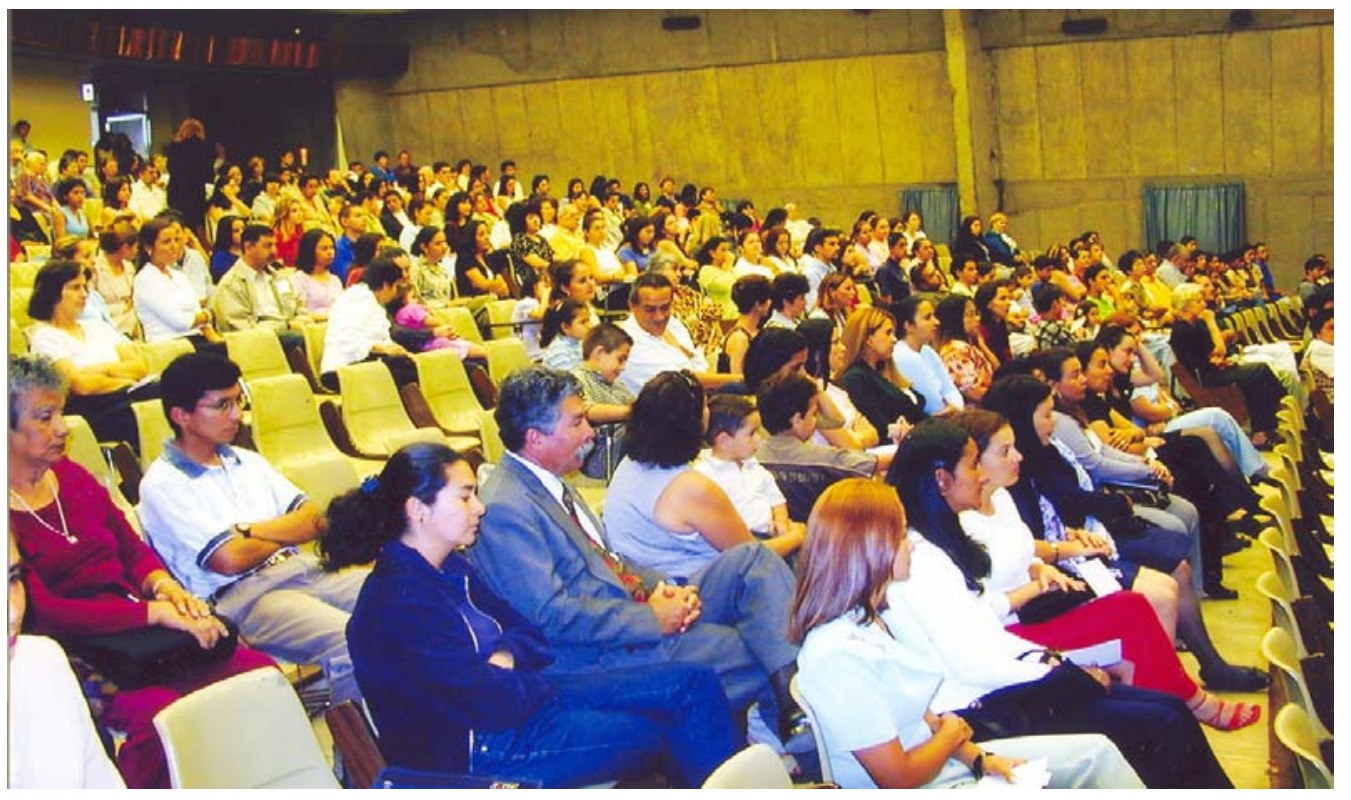

Acto de Clausura de la Capacitación "Encuentro de Matemática Activa y Creativa" 
4.8 Calidad en la Educación: Proyecto de asesoría curricular en la Escuela Bilingüe de Cartago.

Responsable del proyecto: M.Sc. Ana Isabel Mora Vargas.

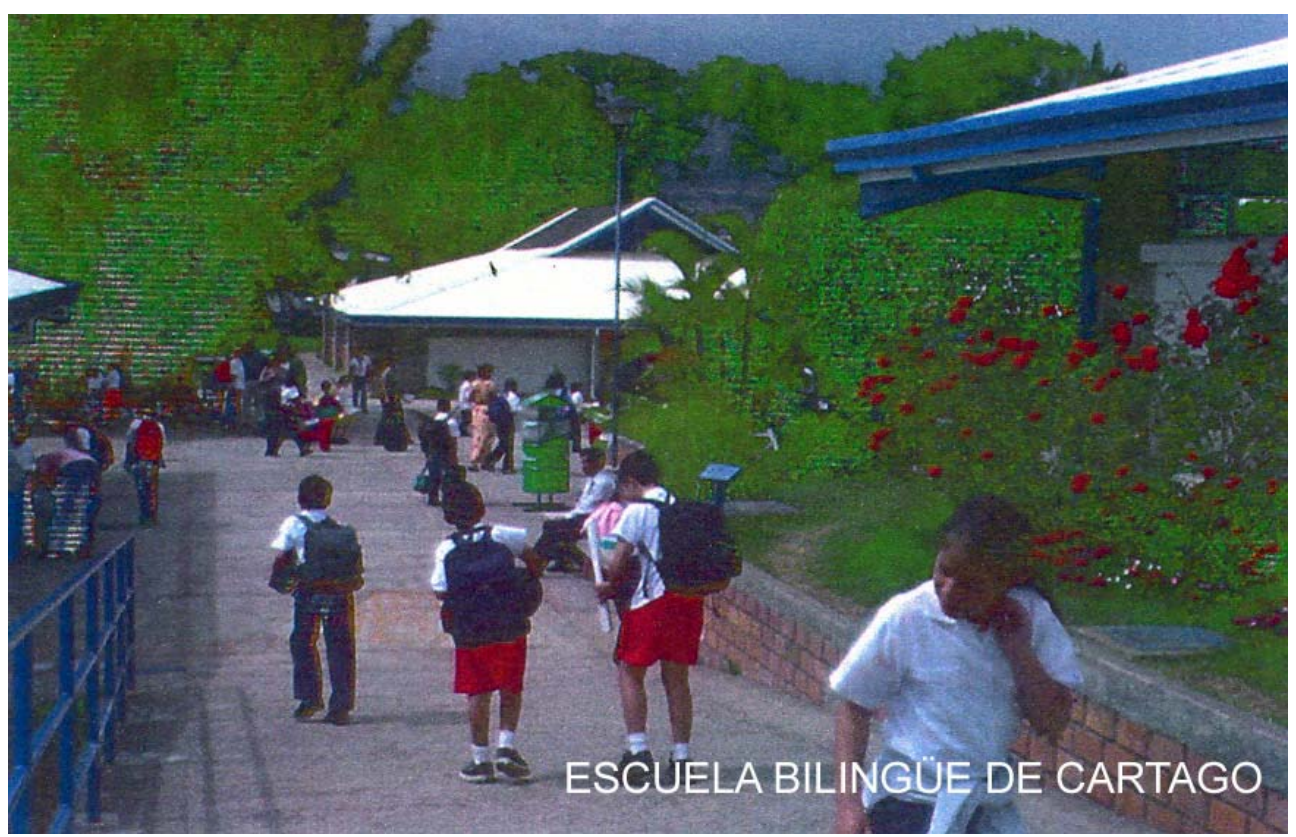

A continuación se realiza un resumen que incluye sus principales logros:

En su primera etapa, y luego de analizar el perfil básico del plan de formación (área académica y área de desarrollo personal) que ofrece la Escuela Bilingüe de Cartago en los niveles de preescolar hasta sexto grado, se determinó:

- La fundamentación del enfoque curricular (perspectivas: filosófica, psicológica, sociocultural y pedagógica).

- Los conocimientos, habilidades, destrezas, valores y actitudes que se pretenden desarrollar en los alumnos y las alumnas del Centro Educativo, en las asignaturas de español, matemáticas e inglés.

- La relación entre las estrategias didácticas de los docentes y las estrategias de estudios de los estudiantes en las asignaturas en estudio (matemáticas, inglés y español) y los recursos didácticos que se utilizan para el desarrollo del plan de formación.

- La congruencia y consistencia entre la fundamentación curricular, los componentes del perfil del docente y las estrategias didácticas. 
- Se determinaron las necesidades de autoformación que tienen los y las docentes de la Escuela.

En la segunda etapa se efectuó un análisis y reflexión del proceso educativo, según el diagnóstico, se analizó y reflexionó respecto a:

- La fundamentación del currículo de la Escuela y su relación con los componentes del área académica y de desarrollo personal.

- Los aspectos positivos y negativos en la planificación, administración y ejecución del currículo.

- Las estrategias didácticas y los criterios de evaluación y su relación con la calidad de la educación.

La tercera etapa corresponde a las propuestas para el mejoramiento de la calidad de su proceso educativo, por lo que se concretaron:

- Lineamientos o estrategias para el mejoramiento de la calidad de la educación en la Escuela Bilingüe de Cartago, en las asignaturas de matemáticas, español e inglés, con base en los resultados obtenidos en la segunda etapa.

- Se valoró el proceso investigativo mediante la devolución de la información a los participantes y la obtención de nuevos aportes.

- Se elaboraron estrategias para las áreas de matemáticas, español e inglés.

4.9 Investigación en el aula: fundamentos teóricos y prácticos.

Responsable de la actividad: Dra. Jacqueline García Fallas.

A solicitud del Colegio de Licenciados y profesores (COLYPRO), la Dra. García Fallas, organizó una capacitación a la que asistieron 19 docentes que laboran en educación secundaria. De acuerdo con los objetivos planteados se logró que los participantes plantearan investigaciones a partir de su quehacer cotidiano. Según el diseño investigativo propuesto, se promovieron reflexiones y comentarios sobre su trabajo de aula, así como el papel de la investigación-acción como herramienta que permite analizar, valorar y propiciar una revisión constante y permanente de su práctica pedagógica. 
Se desarrollaron interesantes trabajos investigativos que promovieron aprendizajes a partir de su propia experiencia de aula, y que finalizaron con productos concretos que favorecieron el trabajo cotidiano de cada docente. Los logros obtenidos por estas personas se resumen de la siguiente manera:

- Aproximación teórica a los enfoques de investigación-acción partiendo del trabajo de aula.

- Integración de diversas características y modelos metodológicos.

- Conocimientos en el diseño de proyectos de investigación.

- Desarrollo de trabajo en equipo en el quehacer investigativo.

- Reflexión analítica y crítica sobre la práctica pedagógica.

- Sistematización de lo cotidiano y presentación de informes.

Cabe indicar la importancia de desarrollar metodologías para promueven aprendizajes mediante procesos investigativos, ya que permiten el logro de conocimientos, actitudes y destrezas, y de esta manera se favorece el trabajo cotidiano de quienes participan en la enseñanza y en el aprendizaje.

\subsection{Red Interinstitucional de la Primera Infancia.}

Responsables de la actividad: Dra. Ana Lupita Chaves Salas, Lic. Jeannette Cerdas Núñez y M.Sc. Kryssia Brade Jiménez.

Es importante mencionar que la Red Interinstitucional de la Primera Infancia se conformó con el fin de propiciar espacios de reflexión y coordinación de acciones entre instituciones gubernamentales y no gubernamentales que promueven la atención integral de la niñez de nuestro país. Inicia sus labores en

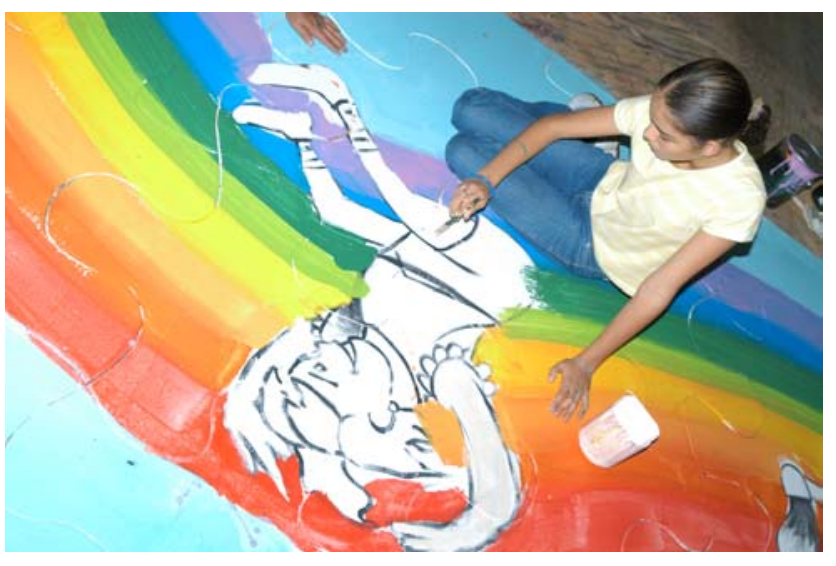
marzo del 2003 con la participación de representantes de UNICEF y de instituciones y universidades estatales, en el marco del Programa Interdisciplinario de Estudios y Acción Social de los derechos de la Niñez y la Adolescencia (PRIDENA) de la Universidad de Costa Rica. 
A continuación se expone un resumen con los principales logros, de acuerdo con los objetivos planteados:

- Se promovió la coordinación interinstitucional para sensibilizar a la población sobre la importancia de la atención integral de la primera infancia.

- En lo que respecta a la divulgación del trabajo que realiza la Red, se diseñaron desplegables y afiches. La UNICEF se encargó de la financiación y publicación de los mismos.

- Se propiciaron acciones interinstitucionales para unificar criterios conceptuales en relación con el tema de la Primera infancia en Costa Rica que sirvan de insumo a una Política Nacional.

- Se organizó el taller denominado, "Estudio Nacional de Creencias y Prácticas de Crianza en Costa Rica", relacionado con los patrones de crianza.

Se ofreció el curso Devinfo, a cargo de la Máster Kryssia Brade de la UNICEF., con el propósito de brindar capacitación para la aplicación de la tecnología Devinfo en Costa Rica.

- Se visibilizaron programas y proyectos institucionalizados sobre la atención integral a la primera infancia.

- Se preparó la solicitud formal de autorización para incorporar referencias de cursos, proyectos e investigaciones en la página Web de la Red.

Queda pendiente darle seguimiento al Convenio de Cooperación Interinstitucional (UNICEF-CONARE).

- Se generaron espacios de reflexión que contribuyeron a mejorar la situación de las niñas y los niños que se encuentran en situación de riesgo durante la primera infancia.

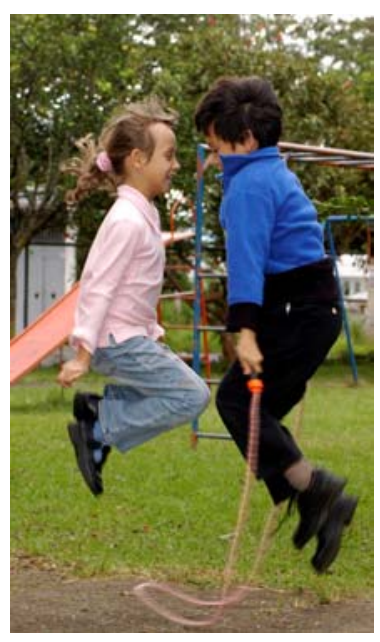


4.11 Procesos Iniciales de Lectura y Escritura desde la filosofía del lenguaje integral. Responsable de organizar la actividad: Dra. Ana Lupita Chaves Salas.

- La Dra. Lupita Chaves S., contactó a la Dra. Ruth Sáez de la Universidad de Río Piedras, Puerto Rico, para que ofreciera una conferencia sobre los procesos iniciales de lectura y escritura bajo el enfoque de la filosofía del lenguaje integral, a docentes y estudiantes de educación preescolar y primaria de la Universidad de Costa Rica y de otras instituciones formadoras de docentes, incluyendo la asistencia

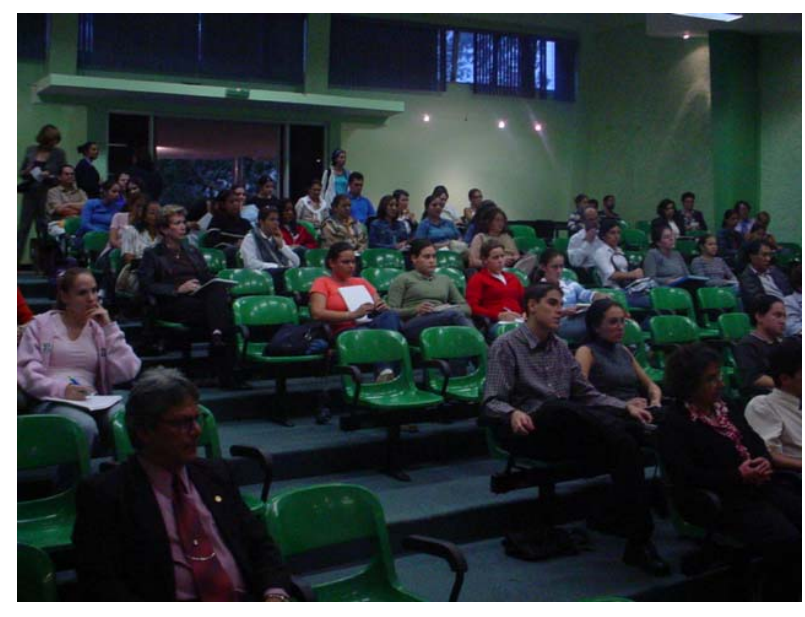
de público en general. Se dieron a conocer resultados de investigaciones recientes en torno a los procesos iniciales de lectura y escritura a partir de la filosofía del Lenguaje Integral, lo que propició una interesante reflexión a partir de esas propuestas investigativas.

- Otro logro importante de mencionar es que la Dra. Sáez ofreció un taller sobre "La filosofía del lenguaje integral", al personal docente de la Escuela Nueva Laboratorio Emma Gamboa, lo que resultó de gran provecho pues permitió un intercambio de conocimientos, experiencias y reflexiones sobre el tema. La actividad permitió el fortalecimiento académico y profesional del personal docente, ya que además beneficia el desarrollo integral de la población infantil de la institución mencionada.

Se espera que el intercambio de experiencias entre la Dra. Sáez y nuestros educadores, haya permitido nuevas formas de incentivar y enseñar bajo innovadoras metodologías del lenguaje integral.

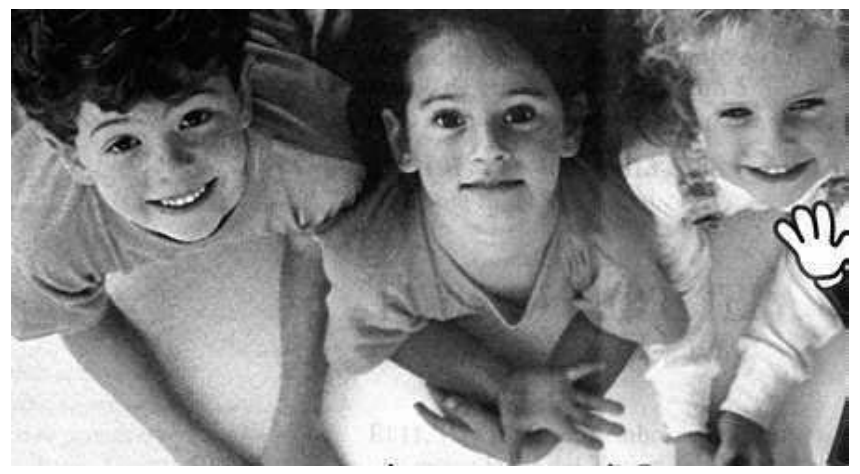


4.12 Kalydria: El espejo de mil rostros, su presentación a la comunidad universitaria y nacional.

Responsables de organizar la actividad: Dra. Ana Lupita Chaves Salas y M.Sc. Julieta Castro Bonilla.

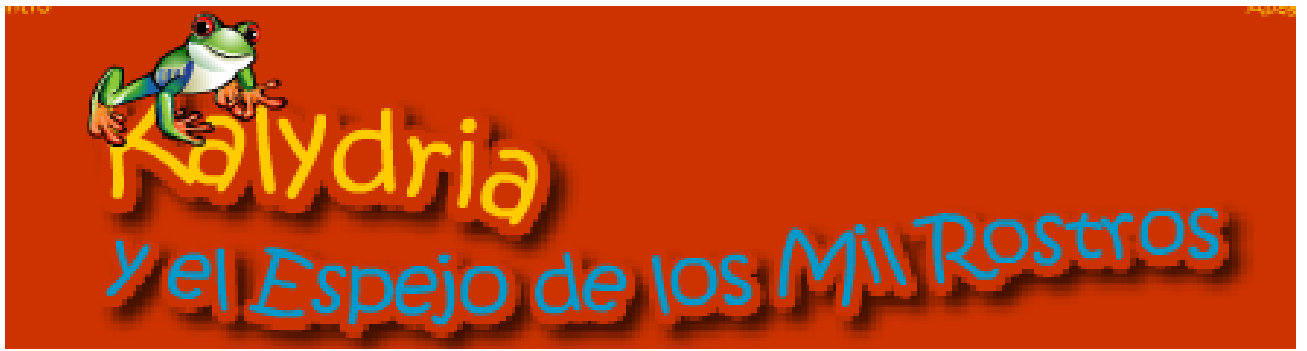

\section{Autoras:}

Dra. Alicia Gurdián Fernández

Dra. Vanessa Fonseca Rodríguez

- Kalydria el Espejo de mil rostros, es considerado un juego interactivo que motiva a docentes de educación primaria y secundaria con énfasis en el área de las ciencias, pues les permite planificar actividades didácticas interactivas como: guías, reflexiones, juegos, adivinanzas y mapas conceptuales, que estimulan la socialización y los aprendizajes significativos. Debido a que este juego interactivo afecta directamente los sentidos, los procesos de enseñanza y aprendizaje en el área ambiental se construyen con mayor facilidad.

- Este juego interactivo hace un llamado de atención hacia los retos que impone la crisis energética ambiental, pues se utiliza el ambiente virtual como recurso de aprendizaje, ya que permite establecer una interacción estrecha entre el hombre y naturaleza.

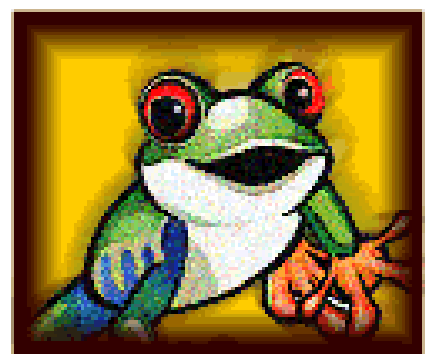

Agalychnis callidryas
- El desarrollo de la actividad permitió compartir el proyecto Kalydria el espejo de mil rostros, el cual representa una innovación que contribuyó a los procesos de formación, capacitación y actualización de los participantes. Se promovieron innovadoras nuevas estrategias y técnicas didácticas que se pueden aplicar como parte de los procesos de enseñanza y 
aprendizaje. El educador debe aprovechar su creatividad y entusiasmo para llevar a la práctica este tipo de recursos didácticos, pues permiten enseñanzas y procesos de conocimiento desde un planteamiento divertido y agradable. El nombre de este sitio es www.kalydria.org

4.13 Presentación a la comunidad nacional y universitaria de la Revista Electrónica: Actualidades Investigativas en Educación.

Responsables de la actividad: Dra. Ana Lupita Chaves Salas y MEd. Julieta Bonilla.

- Se dio a conocer a la comunidad universitaria y nacional la Revista Electrónica "Actualidades Investigativas en Educación", que corresponde a la producción académica que realizan investigadores, docentes y especialistas que laboran en este Instituto, en las unidades académicas que conforman la Universidad de Costa Rica, así como de otras instituciones, tanto dentro como fuera del país. La producción científica presentada respondió a "tres volúmenes con dos números cada uno", correspondiente a los años 2001, 2002 y 2003, para un total de 31 artículos.

- La Revista, es considerada un recurso interactivo para la divulgación de artículos, ya que al poseer un Consejo Editorial, asegura que los artículos sean valorados con criterios de evaluación exigidos por toda revista científica.

Al contar con este respaldo, los artículos califican dentro de los parámetros aceptados por la Comisión de

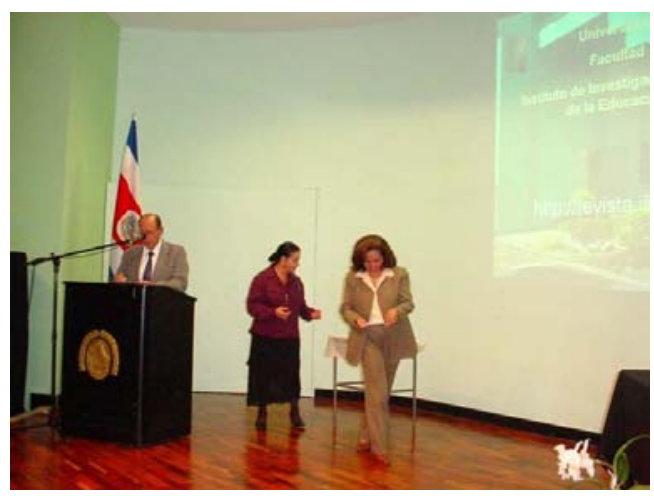
Régimen Académico de la Universidad de Costa Rica.

- Mediante la presentación de los artículos, los participantes tuvieron la oportunidad de conocer la importancia y utilidad que poseen las tecnologías de la información y de la comunicación, ya que permiten el desarrollo de conocimientos, habilidades y destrezas sobre temáticas educativas formales e informales, mediante intercambios virtuales que mejoran los procesos de enseñanza y aprendizaje. 


\section{Consideraciones finales}

Para la dirección del Instituto de Investigación en Educación-INIE, y para la coordinación del Programa Educación Continua y Servicios Especiales, el logro de sus objetivos y metas representan un gran compromiso con la comunidad universitaria y nacional, que compromete al Instituto a continuar trabajando y mejorando el trabajo que realizan sus investigadores.

Como se indicara en el documento, las actividades desarrolladas en el transcurso del año 2004 se organizaron con el apoyo de las coordinadoras de los programas de investigación que conforman el quehacer del Instituto, ya que de manera constante y sistemática se organizan actividades con instituciones y unidades académicas, dentro y fuera de la Universidad, lo que facilita la afluencia de diversos públicos

Además de la concepción filosófica que sustenta el concepto de educación en el Instituto, nuestro trabajo se orienta en función del desarrollo pleno, integral y armonioso de los individuos. Por lo que se considera fundamental participar en una formación humanística que enfatice la solidaridad y la cooperación, el respeto a la diversidad y a los derechos de las personas, así como a las expresiones culturales, una formación que fomente la acción responsable frente a la naturaleza y al entorno social y cultural, y que enfatice las cualidades humanas frente al vertiginoso avance científico y tecnológico.

El documento evidencia el compromiso que tiene la Universidad de Costa Rica y el Instituto de Investigación en Educación-INIE-, a través del programa Educación Continua y Servicios Especiales, con la sociedad costarricense, al generar, producir y transmitir conocimientos a partir de procesos investigativos que fortalecen la educación y por consiguiente el desarrollo individual y colectivo.

Los resultados de sus investigaciones se ofrecen a diversas poblaciones por medio de la planificación y organización de talleres, mesas redondas y conferencias, mediante las cuales se divulgan de los procesos investigativos que se desarrollan en el Instituto. 


\section{Referencias}

Proyecto Estado de la Nación. (2001). Desarrollo sostenible: Módulo para educación secundaria. San José, C.R.: Proyecto Estado de la Nación.

Chaves S., Lupita. (2005). Informe de labores 2004 del Instituto de Investigación en Educación. San José, Costa Rica: INIE.

Dengo, María Eugenia. (2004). Educación Costarricense. San José, Costa Rica: Editorial Universidad Estatal a Distancia.

López A., Olimpia. (2004). La Universidad del Siglo XXI. San José, C.R.: Ediciones Guayacán.

Gurdián F., Alicia. (2000). Programa de Extensión y Educación Permanente del Instituto de Investigaciones para el Mejoramiento de la Educación Costarricense. Sin publicar.

Rojas P., Marta. (2005). INIE, Trayectoria de Calidad y aporte a la educación. En Girasol. Año 8, $\mathrm{N}^{\circ} 26$.

Universidad de Costa Rica, Consejo Universitario. (2001). Estatuto Orgánico. San José, C.R.: El Consejo. 